\title{
Inhibitory effect of dsRNA TLR3 agonist in a rat hepatocellular carcinoma model
}

\author{
YU-YIN XU ${ }^{1,2^{*}}$, LI CHEN $^{1 *}$, JIA-MING ZHOU $^{1}$, YUAN-YUAN WU $^{1}$ and YUAN-YUAN ZHU ${ }^{3}$ \\ ${ }^{1}$ Department of Pathological Anatomy, Nantong University, ${ }^{2}$ Department of Nephrology, \\ Affiliated Hospital of Nantong University, Nantong University, Nantong, Jiangsu 226001; \\ ${ }^{3}$ Biomics Biotechnologies Co., Ltd., Nantong, Jiangsu 226016, P.R. China
}

Received June 14, 2012; Accepted July 31, 2013

DOI: $10.3892 / \mathrm{mmr} .2013 .1646$

\begin{abstract}
Hepatocellular carcinoma (HCC) is one of the most common types of malignant tumor. Studies have demonstrated that the toll-like receptor 3 (TLR3)/interferon pathway is inhibitory in cancer cell proliferation, suggesting that the activation of this pathway may have therapeutic potential. In the present study, the inhibitory effects of BM-06, a double-stranded (ds)RNA TLR3 agonist, against HCC were studied in vivo. Using a 2-acetylaminofluorene-induced HCC rat model, histological examination and analysis of corresponding biomarkers following treatment with BM-06, showed a decrease in tumor growth and cell proliferation, and an increase in apoptosis compared with that in a phosphate-buffered saline control group. In addition, the observed antitumor effect of BM-06 in the HCC rat model was demonstrated to be superior to the known TLR3 agonist, polyinosinic-polycytidylic acid.
\end{abstract}

\section{Introduction}

Hepatocellular carcinoma (HCC) is the most common malignancy of the liver, the seventh most common type of cancer and the third leading cause of cancer-related mortality worldwide (1). The long-term prognosis of patients with HCC remains unsatisfactory due to tumor recurrence and a limited response to chemotherapy and radiotherapy (2-5). A number of treatment options are currently available; however, mechanisms of liver cancer initiation and progression have yet to be adequately elucidated. Therefore, an understanding of the mechanisms of HCC proliferation and metastasis may improve the efficacy of treatment modalities for CLL.

Correspondence to: Professor Li Chen, Department of Pathological Anatomy, Nantong University, 19 Qixiu Road, Nantong, Jiangsu 226001, P.R. China

E-mail: bl1@ntu.edu.cn

${ }^{*}$ Contributed equally

Key words: toll-like receptor 3, double-stranded RNA, hepatocellular carcinoma, apoptosis
Toll-like receptor 3 (TLR3) is a pattern-recognition receptor that is involved in immune signaling and is essential for survival as it recognizes various viral components, such as double-stranded (ds)RNA. TLR3 signaling depends solely on the TIR domain-containing adaptor-inducing interferon- $\beta$ (TRIF) adaptor protein. This leads to the activation of the nuclear factor- $\kappa \mathrm{B}(\mathrm{NF}-\kappa \mathrm{B})$ and interferon regulatory factor 4 (IRF4) transcription factors, which induce an antiviral interferon response (6,7). Furthermore, TRIF exhibits pro-apoptotic activity, suggesting that TLR3 signaling may lead to cell death (8).

Although TLR3 may directly trigger apoptosis in certain cancer cells $(9,10)$, dsRNA has been recognized as a TLR3 ligand and was reported to induce apoptosis in several cell types through multiple pathways. Results of previous studies have indicated that the neoplastic process may disrupt the TLR signaling pathways, thereby allowing for the unhindered progression of cancer. Furthermore, TLRs on tumor cell surfaces facilitate evasion from immune surveillance via the suppression of T-cell proliferation and natural killer cell activity. This suggests that TLR signaling in tumor cells is associated with the progression of cancer and evasion of host defenses. However, the involvement of TLR3 signaling pathways in tumor progression, invasion and metastasis remains unclear.

In the present study, it was hypothesized that dsRNA acts as a TLR3 ligand and may be capable of inhibiting HCC activity in vivo by regulating the TLR3 signaling pathways. In the study, several dsRNA sequences were previously designed and synthesized based on cell surface TLR3 sensitive viral sequences in human echovirus. The expression of TLR3 signaling proteins following stimulation with one particular dsRNA (BM-06) in HCC tissues was investigated. In addition, the correlation between TLR3 activation and cell proliferation or apoptosis in HCC tissues was analyzed using an orthotopic Sprague-Dawley (SD) rat HCC model.

\section{Materials and methods}

Reagents. Polyinosinic-polycytidylic acid [poly(I:C)], a dsRNA, TLR3 specific ligand, was purchased from Invitrogen Life Technologies (Carlsbad, CA, USA). Several dsRNAs were designed based on cell surface TLR3 sensitive viral sequences 
in human echovirus, human poliovirus, enterovirus 70 and coxsackievirus from GenBank. Furthermore, the viral sequences were submitted for basic local alignment search tool (BLAST) analysis (http://www.ncbi.nlm.nih.gov/blast/) to ensure that the sequence was not homologous to human genes. The target sequence of BM-06 was CCGGCCCCUGA AUGCGGCUAAUC (23 nt) and was synthesized by Biomics Biotechnologies Co., Ltd. (Jiangsu, China).

Orthotopic Sprague-Dawley (SD) rat HCC model. Thirty male SD rats, (age, 4-6 weeks; weight, 120-160 g), were used. SD rats were purchased from the animal center of Nantong University (Jiangsu, China) and housed according to the guidelines for experimental animals approved by the Animal Care and Use Committee of Nantong University. All 30 rats were fed with animal feed containing $0.03 \%$ 2-acetylaminofluorene (2-AAF; Sigma-Aldrich, St. Louis, MO, USA) and kept in an air-conditioned environment for 16 weeks to establish the HCC model. Ten rats were sacrificed under mild ether anesthesia at the 12th, 14th, 16th and 18th week after the rats were fed with animal feed containing 2-AAF. Livers were collected to confirm malignant transformation. Fifteen HCC-established rats were randomly selected and divided into three groups of five rats per cage. The remaining five HCC rats were used to replace, if required, any rat that died during the experiment. The study was approved by the Ethics Committee of Nantong University, Jiangsu, China.

Drug treatment. All procedures were conducted in accordance with the guidelines for experimental animals approved by the Animal Care and Use Committee of Nantong University, (Jiangsu, China).

Two of the above animal groups were treated, respectively, with the drug candidate (BM-06) and poly(I:C) which served as a positive control. BM-06 and poly(I:C) were prepared in sterile $0.01 \mathrm{M}$ phosphate-buffered saline (PBS) and injected intraperitoneally at $1.0 \mathrm{mg} / \mathrm{kg}$ once a week for 6 weeks. The third rat group was treated in the same manner; however, the rats were injected with $1.0 \mathrm{mg} / \mathrm{kg}$ PBS to be used as a control group. Treatments were initiated when the rats had been fed with 2-AAF for 16 weeks. At the end of the treatment period, all 15 treated rats were sacrificed and the livers were excised and weighed. Specimens were taken from the liver for pathological examination and immunohistochemical analysis, and the remaining liver samples were stored at $-80^{\circ} \mathrm{C}$ for RNA and protein analyses.

Hematoxylin and eosin $(H \& E)$ staining. Rat liver tissues fixed in a $10 \%$ formalin solution were sectioned (4- $\mu \mathrm{m}$ thickness) following dehydration, being made transparent and paraffin-embedding. The sections were flattened, pasted and heated on glass slides. Histological evaluations were performed by $\mathrm{H} \& \mathrm{E}$ staining and pathological examination $(11,12)$.

Immunohistochemical staining. Rat HCC sections $(4-\mu \mathrm{m}$ thickness) were deparaffinized and hydrated. Antigen retrieval was conducted in a $140 \mathrm{mM}$ citrate buffer $(\mathrm{pH} 6.0)$ in a decloaking chamber (BioCare Medical, Walnut Creek, CA, USA) for $3 \mathrm{~min}$ at $115^{\circ} \mathrm{C}$. The slides were then transferred to boiling de-ionized water and cooled for $20 \mathrm{~min}$ at room
Table I. Primer sequences.

\begin{tabular}{ll}
\hline Primer & \multicolumn{1}{c}{ Sequence $\left(5^{\prime} \rightarrow 3^{\prime}\right)$} \\
\hline R-TLR3-F & CGGTCAAGGTGTTCAAGA \\
R-TLR3-R & GGATGGTAGAAGCGTGTT \\
R-NF-kB-F & TGGCTTCTATTACCTGTA \\
R-NF-kB-R & TAACGACATATACCATCAG \\
R-caspase-8-F & TGAACTATGATGTGAGCAATA \\
R-caspase-8-R & TTCCGTAGTGTGAAGATG \\
R-IFN- $\gamma$-F & TCTTCACATCAAAGGAGTCATC \\
R-IFN- $\gamma$-R & TGCTGCTGGAGGTCATTA \\
R-VEGF-F & GCAGCATAGCAGATGTGAAT \\
R-VEGF-R & TTGACCCTTTCCCTTTCCT \\
R- $\beta$-actin-F & TATGGAATCCTGTGGCATC \\
R- $\beta$-actin -R & GTGTTGGCATAGAGGTCTT
\end{tabular}

TLR3, toll-like receptor 3; NF- $\mathrm{B}$, nuclear factor- $\kappa \mathrm{B}$; IFN- $\gamma$, interferon- $\gamma$; VEGF, vascular endothelial growth factor; $\mathrm{R}$, reverse primer; F, forward primer.

temperature (RT) to block endogenous peroxidase, endogenous biotin and non-specific proteins. The slides were incubated with the following antibodies: anti-survivin (dilution, 1:100; Santa Cruz Biotechnology, Inc., Santa Cruz, CA, USA), -Bcl-2 (dilution, 1:50; Santa Cruz Biotechnology, Inc.), -proliferating cell nuclear antigen (PCNA; dilution, 1:100; Santa Cruz Biotechnology, Inc.) and -caspase-3 (dilution, 1:100; Santa Cruz Biotechnology, Inc.) for $30 \mathrm{~min}$ at RT, and maintained overnight at $4^{\circ} \mathrm{C}$. The slides were subjected to a standard avidin-biotin-peroxidase complex technique. Staining was visualized using a DAB+ substrate chromogen solution and hematoxylin QS counterstain (Vector Laboratories, Burlingame, CA, USA). The sections were analyzed by two independent investigators with knowledge of histopathology. Five fields of view were captured on each section of the harvested tumor. Representative images were selected and shown.

$q P C R$. Total RNA was isolated from liver tissues using TRIzol (Invitrogen Life Technologies). qPCR was performed for TLR3, NF- $\kappa B$, TRIF, caspase-8, interferon (IFN)- $\gamma$ and vascular endothelial growth factor (VEGF) using an ABI Prism ${ }^{\circledR} 7700$ Sequence Detection system (Applied Biosystems, Carlsbad, CA, USA). The cycling conditions for amplification were as follows: denaturation at $95^{\circ} \mathrm{C}$ for $3 \mathrm{~min}$, 35 cycles of $45 \mathrm{sec}$ at $95^{\circ} \mathrm{C}, 45 \mathrm{sec}$ at $60^{\circ} \mathrm{C}$, annealing at $72^{\circ} \mathrm{C}$ for $30 \mathrm{sec}$ and a final extension at $72^{\circ} \mathrm{C}$ for $7 \mathrm{~min}$. The primer sequences are listed in Table I. Gene expression for each rat was normalized to the $\beta$-actin mRNA copies from the same sample.

Western blot analysis. The HCC rat liver tissues were homogenized. Equal quantities of protein were resolved by $10 \%$ sodium dodecyl sulfate-polyacrylamide gel electrophoresis and transferred onto a polyvinylidene difluoride membrane filter (Immobilon, Millipore, Billerica, MA, USA). The membrane was blocked for $2 \mathrm{~h}$ with 5\% non-fat milk in 
A

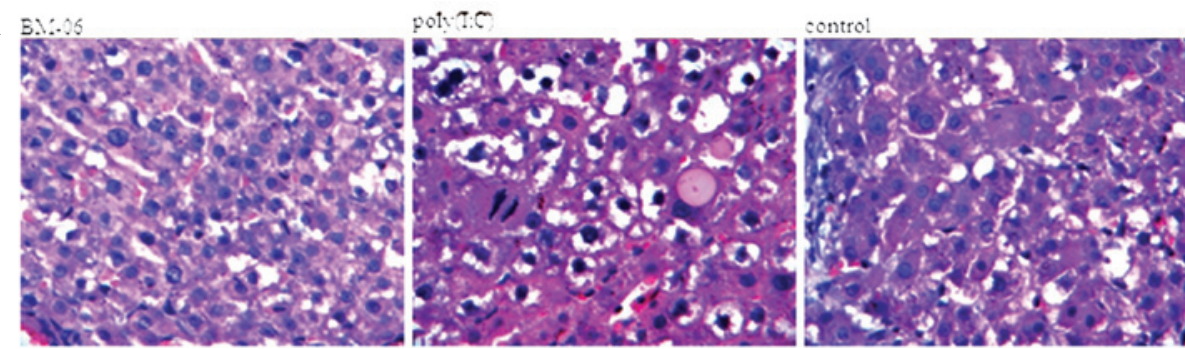

B

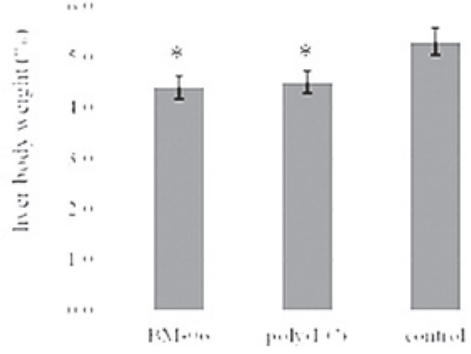

D

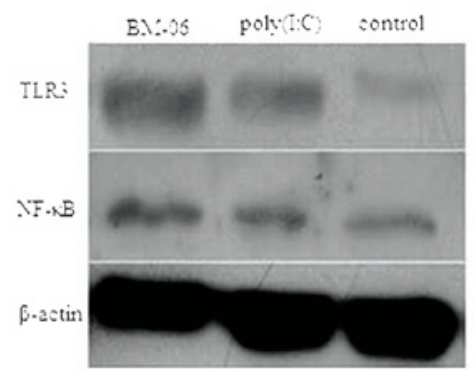

C
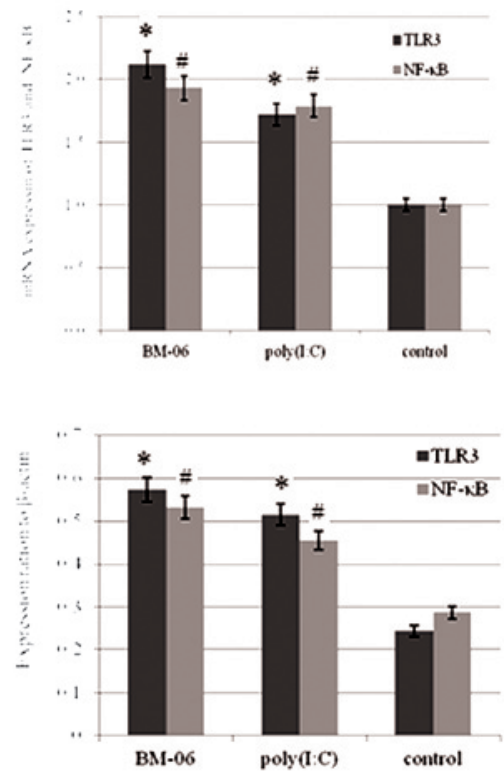

Figure 1. dsRNA-induced inhibition of rat HCC growth. (A) Clear morphological malignant changes in rat HCC livers were observed. The hepatic cells were arranged into funicular form with the nuclei became medium sized, denser chromatin and an increased nucleus to cytoplasm ratio. H\&E staining; magnification, x400. (B) Liver/body weight ratio of treated rats at 23 weeks. Treatment with BM-06 and poly(I:C) led to a reduction in tumor liver weight. ("P $<0.05$ vs. control group). (C) The expression of TLR3 and NF-kB mRNA in the livers of treated rats harvested at 23 weeks was analyzed by qPCR. (D) The expression of toll-like receptor 3 (TLR3) and nuclear factor (NF)-kB protein in the livers of treated rats harvested at 23 weeks was analyzed by western blot analysis. "TLR3, P<0.05 vs. control group; ${ }^{~ N F-\kappa B, ~ P<0.05 ~ v s . ~ c o n t r o l ~ g r o u p . ~ d s R N A, ~ d o u b l e-s t r a n d e d ~ R N A ; ~ H C C, ~ h e p a t o c e l l u l a r ~ c a r c i n o m a ; ~ H \& E, ~}$ hematoxylin and eosin.

Tris-buffered saline containing 0.1\% Tween-20 (TBST), incubated with anti-phospho-NF- $\mathrm{B}$ p65 antibody (dilution, 1:500; Cell Signaling Technology Inc., Danvers, MA, USA) and anti-TLR3 antibody (dilution, 1:500; Abcam, Cambridge, UK), and maintained overnight at $4^{\circ} \mathrm{C}$. The membranes were washed 3 times in TBST for $5 \mathrm{~min}$, incubated with a secondary antibody anti- $\beta$-actin (dilution, 1:2500; Santa Cruz Biotechnology, Inc.) for $2 \mathrm{~h}$ at RT and developed using a chemiluminescence system (Pierce Biotechnology Inc., Rockford, IL, USA). The film was scanned and the density of the bands was measured using ImageQuant software (Molecular Dynamics, Sunnyvale, CA, USA) and expressed as a percentage of the density of the $\beta$-actin band.

Statistical analysis. Statistical analysis was performed using SPSS 17.0 software for Windows (SPSS, Inc., Chicago, IL, USA). The data are presented as the mean \pm standard deviation. Differences between groups were evaluated with analysis of variance (ANOVA) or factorial design ANOVA. $\mathrm{P}<0.05$ was considered to indicate a statistically significant difference. The size of the tumor nodules was quantified using Histolab 5.8 software (Microvision Instruments, Cedex, France).

\section{Results}

Effects of dsRNA-induced inhibition on mouse HCC growth. After being fed with 2-AAF for 16 weeks and treated with BM-06 or poly(I:C) for 6 weeks, the rats were sacrificed. The morphological malignant changes in the rat livers were observed by H\&E staining analysis (Fig. 1A). No lesions were observed in the other organs. This suggested that the dsRNA exhibited no toxic side effects on the other organs of the rats. The liver/body weight ratio, a measure of tumor progression, of the experimental rats was calculated (Fig. 1B). As shown, the liver/body weight ratio in the rats treated with BM-06 and poly(I:C) was significantly decreased compared with the PBS-treated group $(\mathrm{P}<0.05)$. The expression of TLR3, NF- $\kappa \mathrm{B}$ mRNA and protein in the rat liver was investigated using $\mathrm{qPCR}$ and western blot analysis, respectively. Increased expression of TLR3 and NF- $\kappa \mathrm{B}$ was observed in the rat livers treated with BM-06 and poly(I:C), compared with the PBS control group $(\mathrm{P}<0.05$; Fig. $1 \mathrm{C}$ and 1D).

Inhibition of cell proliferation and induction of cell apoptosis in $H C C$. Sections of the livers from the treated groups were immunohistochemically stained for laminin, survivin, Bcl-2 
A

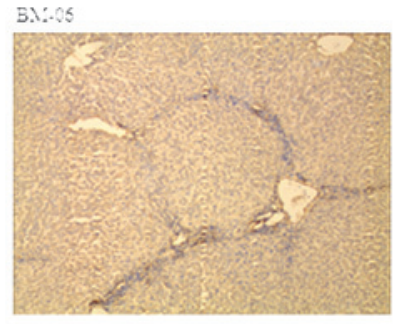

B

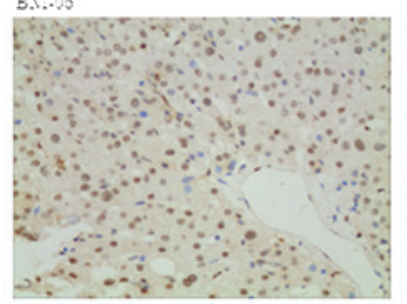

1 .แแนแแ

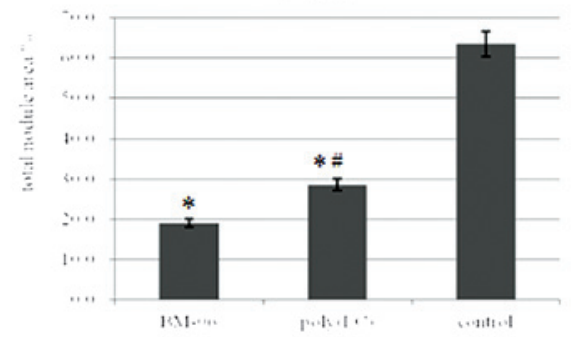

poly:ce

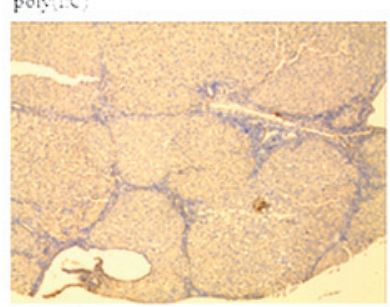

polytic

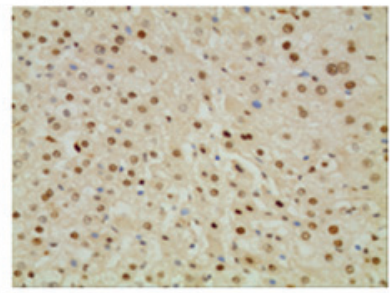

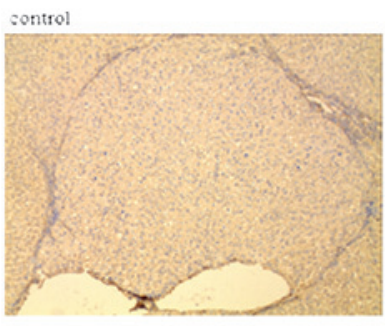

control

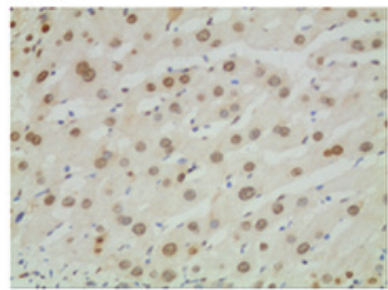

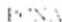

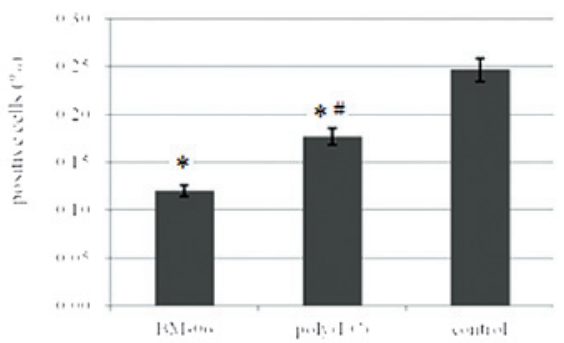

Figure 2. Treatment with BM-06 inhibited proliferation in the rat hepatocellular carcinoma (HCC) model. (A) Sections of livers of treated rats harvested at 23 weeks were stained with anti-laminin antibody to visualize tumor nodules. Nodule size (five mice per treatment group) was quantified using Histolab 5.8 software (immunohistochemistry staining; magnification, x100). (B) Sections of livers of treated rats harvested at 23 weeks were stained for proliferating cell nuclear antigen (PCNA), a proliferation marker (immunohistochemistry staining; magnification, $\mathrm{x} 400$ ). ${ }^{*} \mathrm{P}<0.05$ vs. control group; ${ }^{\#} \mathrm{P}<0.05$ vs. BM-06 group.

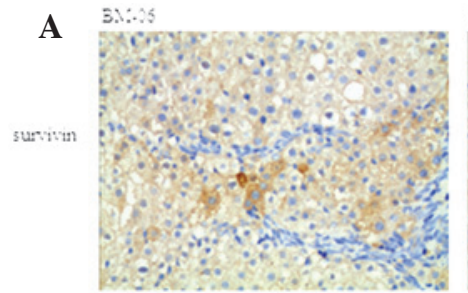

B
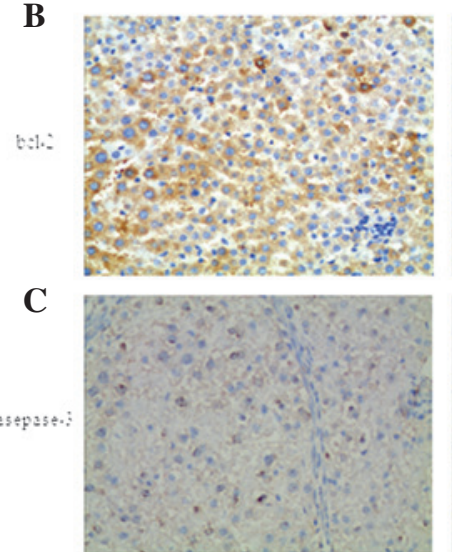

poly to
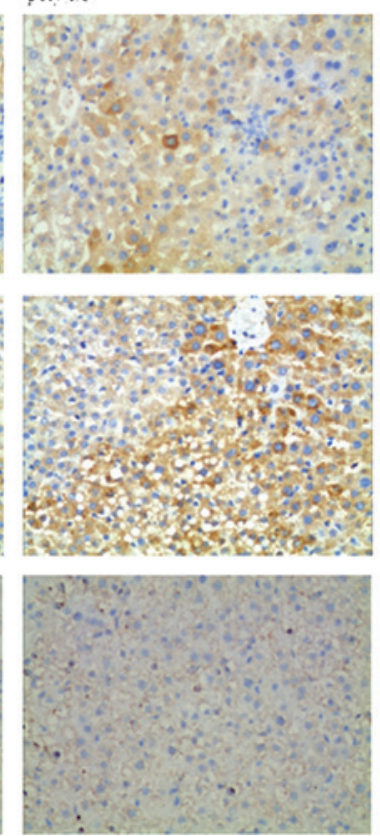

sontro!
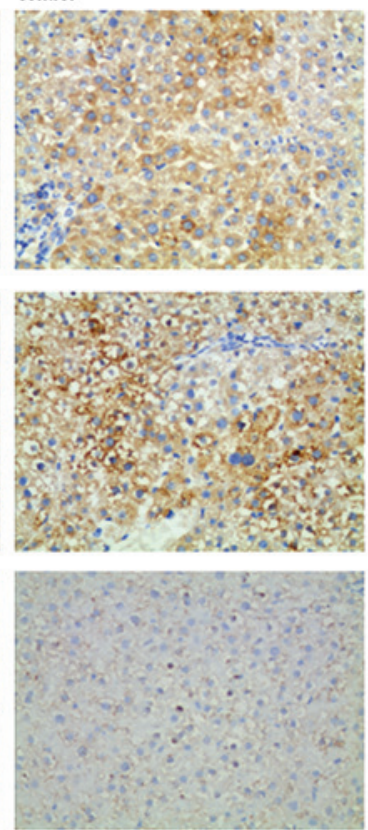

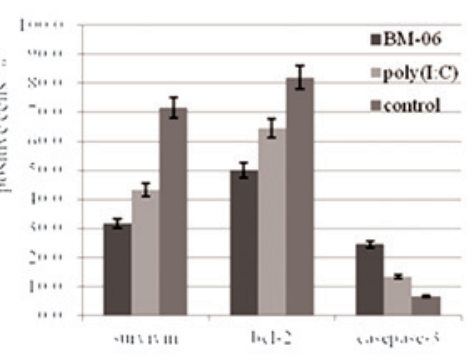

เแกเม

Figure 3. Treatment with BM-06 induced cell apoptosis in the rat hepatocellular carcinoma (HCC) model. Double-stranded (ds)RNA induced HCC cell apoptosis. Reduced numbers of (A) survivin- and (B) Bcl-2-positive cells and increased numbers of (C) caspase-3-positive cells were detected in the livers of rats treated with dsRNA (immunohistochemistry staining; magnification, $\mathrm{x} 400$ ). ${ }^{*} \mathrm{P}<0.05$ vs. control group; ${ }^{\text {"P }} \mathrm{P}<0.05$ vs. $\mathrm{BM}-06$ group.

(two anti-apoptosis markers), caspase-3 (a pro-apoptosis marker) and PCNA (a proliferation marker). As capillarization of the blood supply in the mouse HCC was accompanied by the upregulation of laminin expression within the tumor nodules (13), laminin immunostaining was used to visualize tumor nodules in the liver sections of the treated rats and 


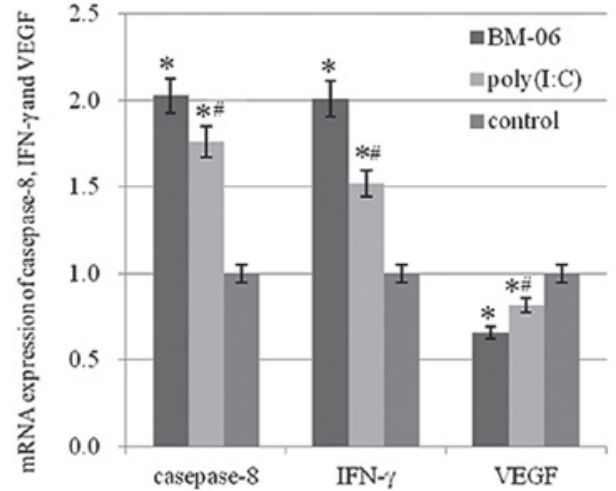

Figure 4. Gene expression of caspase-8, IFN- $\gamma$ and vascular endothelial growth factor (VEGF) in liver tissues of treated rats harvested at 23 weeks was analyzed. The $\mathrm{y}$-axis indicates normalized expression values. ${ }^{*} \mathrm{P}<0.05$ vs. control group; ${ }^{\#} \mathrm{P}<0.05$ vs. BM-06 group.

to determine their sizes with Histolab software (Fig. 2A). Treatment with BM-06 and poly(I:C) was shown to lead to a reduction in the tumor nodule size compared with that in PBS-treated animals, particularly for the BM-06 treated group $(\mathrm{P}<0.05)$. In addition, immunohistochemical staining for positive cells was identified in five randomly selected microscopic fields on sections of five different liver specimens per treatment group. The number of PCNA-, survivin- and Bcl-2-positive cells was significantly reduced, while the number of caspase-3 positive cells was significantly increased in the BM-06 and poly(I:C) groups, compared with that in the PBS control group $(\mathrm{P}<0.05)$. Representative micrographs are shown in Figs. 2 and 3 together with quantification results expressed as a proportion of positive cells.

Increase in mRNA levels of caspase-8 and IFN- $\gamma$, and a decrease in VEGF $m R N A$. mRNA levels of caspase- 8 , IFN- $\gamma$ and VEGF in the liver tissues of the treated rats were determined by qPCR. The results demonstrated that the mRNA levels of caspase- 8 and IFN- $\gamma$ were significantly increased in the liver, while VEGF mRNA levels were significantly decreased in the BM-06 and poly(I:C) group compared with that of the PBS control group $(\mathrm{P}<0.05)$. The effect of BM-06 was greater than poly $(\mathrm{I}: \mathrm{C})(\mathrm{P}<0.05$; Fig. 4$)$. The results suggested that the inhibitory effects of BM-06 may be due to the activation of an innate interferon response and via the initiation of the death-receptor pathway of cell apoptosis in the rat HCC model.

\section{Discussion}

HCC is a complex disease with the involvement of multiple signaling pathways in its pathogenesis and is difficult to treat, particularly in advanced stages. Inhibition of specific growth factor receptors and their various signaling pathways via targeted therapy appears to be a promising approach in the treatment of HCC. More advanced studies are required to fully clarify its molecular pathogenesis and to identify potential key targets for intervention (14).

Recent studies have suggested that dsRNA triggers TLR signaling via MyD88, leading to the induction of NF- $\kappa \mathrm{B}$ and secretion of inflammatory cytokines that prime liver regeneration. TLR3 is unique among TLRs, in that it signals through TRIF but not through MyD88, an event that may lead to activation of either the inflammatory or apoptotic pathway. The inflammatory pathway leads to $\mathrm{NF}-\kappa \mathrm{B}$ activation, while the apoptotic pathway, considered to be mediated by Rip3 binding to TRIF and the recruitment of Fas-associated death domain protein (FADD), leads to caspase-8 activation (14-16).

In a study by Bergé et al (15), it was demonstrated that treatment of HCC mice with poly(I:C) resulted in the suppression of vasculature remodeling and tumor liver growth. It was also observed that the levels of INF- $\gamma$ in the liver were significantly increased in mice treated with dsRNA. INF- $\gamma$ was identified as an inhibitor of endothelial cell proliferation and a potent suppressor of tumor-associated neovascularization. Furthermore, it was suggested that INF- $\gamma$, detected in mouse HCC liver extracts, was released by circulating or resident immune cells. Previous studies have shown that in breast cancer cells, synthetic dsRNA induced apoptosis in a TLR-dependent manner (17). In melanoma cells, TLR3 agonists were able to directly inhibit cell proliferation and induce tumor cell death (9). Zorde-Khvalevsky et al (16) demonstrated that during the initial phase following partial hepatectomy, TLR3 signaling was induced in hepatocytes, leading to the activation of $\mathrm{NF}-\kappa \mathrm{B}$ as well as increased Rip3 protein levels and caspase- 8 activation. Furthermore, NF- $\kappa \mathrm{B}$ was observed to induce pro-IL-1 $\beta$ expression in hepatocytes, which was then activated by caspase- 8 , leading to the inhibition of hepatocyte proliferation.

In the present study, BM-06, a 23-nt dsRNA was designed prior to the experiments and was used as a drug candidate in the rat HCC model. The results showed that BM-06, a TLR3 agonist, stimulated TLR3 on the cell surface and activated $\mathrm{NF}-\kappa \mathrm{B}$. To the best of our knowledge, this study demonstrated for the first time, that the activation of TLR3 inhibited the growth of liver tumors and induced apoptosis of tumor cells in the rat HCC model. The levels of INF- $\gamma$, caspase- 8 and caspase-3-positive cells in the liver were significantly increased, while the levels of VEGF, PCNA-, survivin- and Bcl-2-positive cells were significantly decreased in rat HCCs treated with the dsRNA. It was suggested that the TLR3dependent activation of $\mathrm{NF}-\kappa \mathrm{B}$ in hepatocytes results in an increase in the expression of caspase- 8 and -3 , which subsequently inhibits hepatocyte proliferation and induces HCC cell apoptosis with the recruitment of FADD via caspase- 8 to activity caspase-3. These results demonstrated that the dsRNA inhibited tumor growth through the TLR3 pathway. In addition, BM-06 increased the expression of TLR3. Activation of TLR3 results in the production of IFN- $\gamma$, which may further upregulate the expression of TLR3. In the present study, TLR3 activation by viruses or dsRNA increased the inflammatory potential of the HCC cells, including increased IL-6 production, and further increased IFN- and TGF- $\beta$-responsive gene expression. IFNs may then increase the expression of a number of molecules on the HCC cells, including TLR3. The observations suggested that IFN- $\gamma$ increased the inflammatory potential of the HCC cells by the upregulation of TLR3 and its downstream responses.

Kleinman et al (18) demonstrated that unlike 21- or 23-nucleotide Luc siRNA, 7-, 13-, 16- and 19-nucleotide 
versions did not suppress choroidal neovascularization (CNV). This observation, coupled with data that longer duplexes including 1,000-nucleotide dsRNA and poly(I:C) suppressed CNV, suggested that at least 21 nucleotides are required to activate TLR3. In this study, the length of the dsRNA BM-06 was 23 nt and it achieved TLR3 activation. BM-06, a 23-nucleotide dsRNA, was determined to be superior to poly $(\mathrm{I}: \mathrm{C})$ in the inhibition of $\mathrm{HCC}$ growth and elevated apoptosis of HCC cells with reduced side effects. These results suggested that stimulation of the TLR3 pathway by dsRNA may be sequence-specific.

In conclusion, BM-06 was shown to inhibit HCC activity in vivo. Stimulation of functional TLR3 expressed on rat HCCs by BM-06 activated NF- $\mathrm{B}$ cascade responses, which effectively inhibited the proliferation and growth of HCC in vivo and promoted the apoptosis of HCC cells by initiating the death-receptor pathway of cell apoptosis. Such an effect on the TLR3 by BM-06 was observed to be greater than that of poly(I:C). A greater understanding of the target specific and immunostimulatory function of designed dsRNA as a therapeutic candidate is required for the future development of dsRNA-based treatments for HCC.

\section{Acknowledgements}

This study was supported by funding from the Production-Study-Research Prospective Joint Research Programs of Jiangsu (BY2013042-06), the Priority Academic Program Development of Jiangsu HigherEducation Institutions, the Foundation of the Ministry of Health, Jiangsu, P.R. China (grant no. H201052), the Science Foundation of Nantong City, Jiangsu, P.R. China (grant nos. K2009060 and S2010018) and the Advanced Project of Nantong University. The authors would like to thank Biomics Biotechnologies Co., Ltd., (Jiangsu, China) for aiding with the synthesis of the dsRNA.

\section{References}

1. Yang JD and Roberts LR: Hepatocellular carcinoma: A global view. Nat Rev Gastroenterol Hepatol 7: 448-458, 2010.

2. Nerenstone SR, Ihde DC and Friedman MA: Clinical trials in primary hepatocellular carcinoma: current status and future directions. Cancer Treat Rev 15: 1-31, 1988.
3. Mathurin P, Rixe O, Carbonell N, Bernard B, Cluzel P, Bellin MF, Khayat D, Opolon P and Poynard T: Review article: Overview of medical treatments in unresectable hepatocellular carcinoma - an impossible meta-analysis? (Review) Aliment Pharmacol Ther 12: 111-126, 1998.

4. Llovet JM, Burroughs A and Bruix J: Hepatocellular carcinoma. Lancet 362: 1907-1917, 2003.

5. Kawano Y,Sasaki A, Kai S, Endo Y, Iwaki K, Uchida H, Shibata K, Ohta M and Kitano S: Prognosis of patients with intrahepatic recurrence after hepatic resection for hepatocellular carcinoma: a retrospective study. Eur J Surg Oncol 35: 174-179, 2009.

6. Yamamoto M, Sato S, Hemmi H, Hoshino K, Kaisho T, Sanjo H, Takeuchi O, Sugiyama M, Okabe M, Takeda K and Akira S: Role of adaptor TRIF in the MyD88-independent toll-like receptor signaling pathway. Science 301: 640-643, 2003.

7. Alexopoulou L, Holt AC, Medzhitov R and Flavell RA: Recognition of double-stranded RNA and activation of NFkappaB by Toll-like receptor 3: Nature 413: 732-738, 2001.

8. Heylbroeck C, Balachandran S, Servant MJ, DeLuca C, Barber GN, Lin R and Hiscott J: The IRF-3 transcription factor mediates Sendai virus-induced apoptosis. J Virol 74: 3781-3792, 2000.

9. Salaun B, Coste I, Rissoan MC, Lebecque SJ and Renno T: TLR3 can directly trigger apoptosis in human cancer cells. J Immunol 176: 4894-4901, 2006.

10. Salaun B, Lebecque S, Matikainen S, Rimoldi D and Romero P: Toll-like receptor 3 expressed by melanoma cells as a target for therapy? Clin Cancer Res 13: 4565-4574, 2007.

11. Qun E, Li Chen, Guo Hua Jin, et al: portfolio tool of paraffin tissue microarray[Z]. CN201402229: Nantong university, 2010.

12. Qun E, Li Chen, Qiu Kai E: preparation methods of paraffin tissue microarray[Z]. CN101319971: Nantong university, 2008.

13. Khvalevsky E, Rivkin L, Rachmilewitz J, Galun E and Giladi H: TLR3 signaling in a hepatoma cell line is skewed towards apoptosis. J Cell Biochem 100: 1301-1312, 2007.

14. Chua CW and Choo SP: Targeted therapy in hepatocellular carcinoma. Int J Hepatol 2011: 348297, 2011.

15. Bergé M, Bonnin P, Sulpice E, Vilar J, Allanic D, Silvestre JS, Lévy BI, Tucker GC, Tobelem G and Merkulova-Rainon T: Small interfering RNAs induce target-independent inhibition of tumor growth and vasculature remodeling in a mouse model of hepatocellular carcinoma. Am J Pathol 177: 3192-3201, 2010.

16. Zorde-Khvalevsky E, Abramovitch R, Barash H, Spivak-Pohis I, Rivkin L, Rachmilewitz J, Galun E and Giladi H: Toll-like receptor 3 signaling attenuates liver regeneration. Hepatology 50: 198-206, 2009.

17. Ferreon JC, Ferreon AC, Li K and Lemon SM: Molecular determinants of TRIF proteolysis mediated by the hepatitis $\mathrm{C}$ virus NS3/4A protease: J Biol Chem 280: 20483-20492, 2005.

18. Kleinman ME, Yamada K, Takeda A, Chandrasekaran V, Nozaki M, Baffi JZ, Albuquerque RJ, Yamasaki S, Itaya M, Pan Y, et al: Sequence- and target-independent angiogenesis suppression by siRNA via TLR3. Nature 452: 591-597, 2008. 